\title{
Driven one-component plasmas
}

\author{
Felipe B. Rizzato, ${ }^{*}$ Renato Pakter, ${ }^{\dagger}$ and Yan Levin ${ }^{*}$ \\ Instituto de Física, Universidade Federal do Rio Grande do Sul, Caixa Postal 15051, Porto Alegre 91501-970, RS, Brazil
}

(Received 30 March 2009; revised manuscript received 10 June 2009; published 14 August 2009)

\begin{abstract}
A statistical theory is presented that allows the calculation of the stationary state achieved by a driven one-component plasma after a process of collisionless relaxation. The stationary Vlasov equation with appropriate boundary conditions is reduced to an ordinary differential equation, which is then solved numerically. The solution is then compared with the molecular-dynamics simulation. A perfect agreement is found between the theory and the simulations. The full current-voltage phase diagram is constructed.
\end{abstract}

DOI: 10.1103/PhysRevE.80.021109

PACS number(s): 05.70.Ln, 05.20.-y, 41.85.Ja, 52.25.Dg

\section{INTRODUCTION}

Unlike the equilibrium thermodynamics and statistical mechanics, which are well developed after the pioneering works of Boltzmann and Gibbs, our understanding of nonequilibrium thermodynamics is restricted to some special models and cases. Stochastic lattice gases have provided a fertile testing ground for studying nonequilibrium stationary states in driven systems [1-3]. These models exhibit a variety of phase transition arising from a diffusive (collisional) relaxation. For some of these models local equilibrium and hydrodynamic equations have been derived rigorously [4].

There are, however, other physical systems for which the approach to final stationary state is through a process of collisionless relaxation [5-12]. Gravitational systems and confined one-component plasmas are just two such examples. For these systems the collision duration time diverges and the relaxation is governed by the collisionless Boltzmann (Vlasov) equation [13]. In the thermodynamic limit, the collisionless relaxation process leads to non-MaxwellBoltzmann velocity distributions, even for stationary states without macroscopic currents. Unlike normal thermodynamic equilibrium, the stationary state that follows the collisionless relaxation depends explicitly on the initial distribution of particle positions and velocities. In spite of this complication, it was recently shown that it is possible to construct a statistical theory that quantitatively describes these states $[11,12]$.

Beams of electrons driven by accelerating vacuum devices, such as the thermionic valves, diodes, and magnetrons, also do not relax to the Maxwell-Boltzmann distribution [14]. Unlike the driven stochastic lattice gases, these systems, however, are intrinsically collisionless. An important practical question concerns the kinetic temperature distribution in thermionic devices in which the directed velocity produced by the electric field is comparable to the thermal velocity [15]. This is particularly the case for the transitional region between Child-Langmuir and no-cutoff regimes in magnetrons, where the electric potential becomes comparable to the thermal energy [15]. Even when the final di-

\footnotetext{
*rizzato@if.ufrgs.br

†pakter@if.ufrgs.br

†levin@if.ufrgs.br
}

rected velocity is larger than the thermal velocity, there is a region near the emitting cathode where thermal effects are important. It is of great practical interest to determine the extent of these regions $[16,17]$. Furthermore, since in these systems the collision duration time diverges, there is no local equilibrium, and one cannot a priori postulate an equation of state relating the beam density and the beam temperature, as for adiabatic or isothermal processes [15]. Instead, given the properties of thermionic filaments—such as say the velocity distribution of the emitted electrons-one should solve the boundary-value problem posed by the Vlasov equation. This is the approach used in the present paper. Taking into account the collisionless aspects of the system, we obtain a solution without any assumptions on possible equations of state. Our methodology resembles the one introduced by Alfvén in the context of electric double layers in plasmas [18-20]. To test the predictions of the theory we have developed a moleculardynamics simulation method, which explicitly accounts for the boundary conditions of the system. Excellent agreement is found between the simulations and theory. In particular, our results show that the isothermal hypothesis occasionally used to simplify the analysis of driven one-component plasmas should not be employed.

In Sec. II we present the model and discuss the simulational approach. In Sec. III we compare the solution of the stationary Vlasov equation and results of simulations. In Sec. IV we give our conclusions.

\section{MODEL}

\section{A. Vlasov equation}

As a prototype of a collisionless driven system, we consider a beam of electrons, accelerated by an external electric field, traveling from an emitting (planar) cathode to a collecting (planar) anode across the device gap. The cathode, located at position $x=0$, is kept at electrostatic potential $\varphi(x$ $=0$ ) $=0$ and is heated to temperature $T_{c}$, resulting in the emission of electrons. After traversing the device gap, these electrons are collected at the cold anode $\left(T_{a} \approx 0\right)$ located at $x$ $=L$ and kept at potential $\varphi(x=L)=V>0$. During the steadystate operation, the region between the cathode and anode contains a total of $N$ electrons, resulting in a current density $j$. Our goal is to relate $j$ to the potential difference $V$, the number of electrons $N$, the device width $L$, and the cathode temperature $T_{c}$. 
For planar electrodes, particle distribution transverse to the $x$ axis can be taken to be uniform. Furthermore, the oneparticle distribution function for a collisionless system in a steady state must satisfy the stationary Vlasov equation,

$$
v \frac{\partial f}{\partial x}+\frac{e}{m} \nabla \varphi(x) \frac{\partial f}{\partial v}=0,
$$

where $e$ is the elementary charge, $m$ is the electron mass, and $f=f(x, v)$ is the static distribution function. In the thermodynamic limit, Vlasov equation becomes exact for particles interacting by long-range potentials [21].

It can be readily seen that the distribution functions of the form $f(x, v)=f[\varepsilon(x, v)]$, where $\varepsilon$ is the mean particle energy, $\varepsilon \equiv m v^{2} / 2-e \varphi(x)$, satisfy Eq. (1). Therefore, if $f(\varepsilon)$ is specified at $x=0, f$ is then also determined for any other position, provided that the electrostatic potential $\varphi(x)$ is known. This potential can, in turn, be calculated self-consistently from the solution of the Poisson equation

$$
\frac{d^{2} \varphi(x)}{d x^{2}}=\frac{e}{\epsilon_{0}} n(x),
$$

where the particle density $n(x)$ is given by $n(x)=\int_{v} f(\varepsilon) d v$ $=\int_{v} f\left[m v^{2} / 2-e \varphi(x)\right] d v$, the total particle number is $N$ $=A \int_{v} \int_{0}^{L} f d x d v$, and the transverse cross sectional area of the essentially one-dimensional (1D) device is $A$. To represent both the thermal distribution near the cathode, and the fact that only particles with positive velocities actually move into the device gap, we choose at $x=0$ a unidirectional Maxwellian distribution of the form

$$
f(x=0, v)=\left\{\begin{array}{l}
n_{0} \sqrt{\frac{2 m}{\pi k_{B} T_{c}}} \exp \left(-\frac{m v^{2}}{2 k_{B} T_{c}}\right) \quad \text { if } v \geq 0, \\
0 \quad \text { if } v<0
\end{array}\right.
$$

where $k_{B}$ is the Boltzmann constant, $T_{c}$ is the cathode temperature, and $n_{0}$ is the beam density at the cathode after the stationary state is achieved. The value of $n_{0}$ can only be obtained once the full problem has been resolved. The distribution function over the length of the whole diode is then

$$
f(x, v)=\left\{\begin{array}{l}
n_{0} \sqrt{\frac{2 m}{\pi k_{B} T_{c}}} \exp \left(-\frac{\varepsilon}{k_{B} T_{c}}\right) \text { if } v \geq v_{\text {min }}(x), \\
0 \text { if } v<v_{\text {min }}(x),
\end{array}\right.
$$

where $v_{\min }(x)=\sqrt{\frac{2 e \varphi(x)}{m}}$. The unidirectional velocity distribution is similar to the one used in the analysis of current-free globally neutral double layers [20]; however, in our case we do have circulating current generated by a one-component non-neutral electron beam.

Integrating the distribution function $f[\varepsilon(x, v)]$ over the possible values of velocity, we arrive at a nonlinear integrodifferential equation for the electrostatic potential,

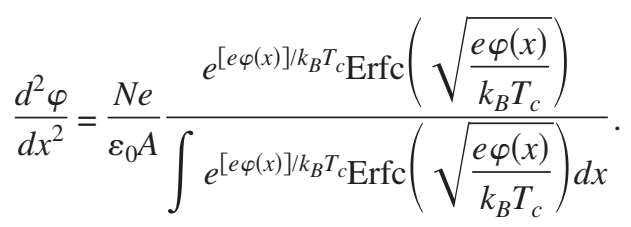

It is important to note the difference between this equation and the Poisson-Boltzmann equation obtained for usual collisional plasmas and electrolytes in the mean-field limit [22]. Equation (5) can be solved numerically to yield the electrostatic potential and the distribution function for the electron beam in a stationary state.

\section{B. Simulations}

For systems with long-range interactions, Vlasov equation should become exact in the thermodynamic limit. To confirm this for our system, we have performed molecular-dynamics simulation of an equivalent one-dimensional model. The simulated system consists of $N_{s}$ mutually interacting charged sheets of area $A$-each containing $n_{s}$ electrons of the same velocity-moving along the $x$ axis, under the action of the external electric field produced by the grounded cathode $\varphi(0)=0$ and an anode kept at a fixed potential $\varphi(L)=V$. The interaction potential between the two sheets $G\left(x_{i}, x_{j}\right)$ is the Green's function [23] of the Laplace equation, $d^{2} G(x, y) / d x^{2}=1 / L \delta(x-y)$ with the boundary conditions $G(x=0, y)=G(x=L, y)=0$. Solving this equation we obtain $G\left(x_{i}, x_{j}\right)=x_{<} / L\left(x_{>} / L-1\right)$, where $x_{<}$and $x_{>}$are the smaller and the larger of the two-particle coordinates $x_{i}$ and $x_{j}$. The effective Hamiltonian for the sheet dynamics is then

$$
H=\sum_{i}\left(\frac{m_{s} v_{i}^{2}}{2}-\frac{e_{s} V}{L} x_{i}\right)-\frac{1}{2} \frac{e_{s}^{2} L}{\varepsilon_{0} A} \sum_{i, j} G\left(x_{i}, x_{j}\right),
$$

where $e_{s}=n_{s} e$ and $m_{s}=n_{s} m$ are the charge and mass of each sheet, respectively. The acceleration of each simulated sheet then follows from the canonical equations of motion,

$$
\dot{v}=\frac{e V}{m L}+\frac{N e^{2}}{2 \varepsilon_{0} m A}\left[\left(\frac{n^{[l e f t]}-n^{[r i g h t]}}{N_{s}}\right)-\left(1-2 \frac{\bar{x}}{L}\right)\right],
$$

where $n^{[l e f t(r i g h t)]}$ is the number of sheets to the left (right) of the one considered, and $\bar{x}$ denotes the positional average $\bar{x}$ $=\sum_{j} x_{j} / N_{s}$. Since $0 \leq \bar{x} \leq L$, from Eq. (7) one sees that the electron acceleration at the device entrance where $n^{[l e f t]} \rightarrow 0$ and $\quad n^{[r i g h t]} \rightarrow N_{s}$ satisfies $e V / m L-N e^{2} / 2 \varepsilon_{0} m A<\dot{v}(x=0)$ $<e V / m L$, which reveals that in space-charge dominated devices where $e V / m L<N e^{2} / 2 \varepsilon_{0} m A$, acceleration at beam entrance may be zero or even negative [24]. When the acceleration vanishes, the associated current is denoted as the limiting one. Since we wish to describe a hot cathode and a cycling current inside the device, we adopt the following strategy. We advance the simulation in small time steps, always obeying Eq. (7). Whenever a particle crosses the anode and exits the system, it is reinjected at the cathode position. At this point all the particles in a small region $\delta_{s}$ around the cathode are rethermalized so as to ensure that the distribution there keeps its original form of a truncated Maxwellian. The width $\delta_{s}$ must be sufficiently small, $\delta_{s} \ll L$, but apart from 

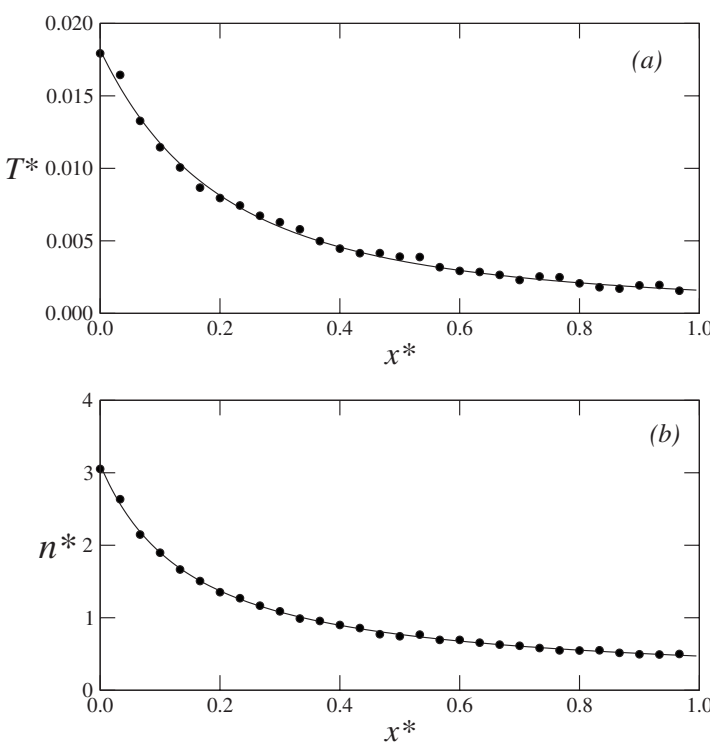

FIG. 1. (a) Temperature and (b) density versus position in the case of the limiting current and $T_{c}^{*}=0.05$. Solid lines represent the theoretical results while the circles are the results of the simulations.

this condition its precise value is arbitrary. The simulations were performed with $\delta_{s} / L=0.01$ and $N_{s}=50000$. In all cases we start with uniform distributions of sheets and compute the observables only after the system reaches its final stationary state. It is interesting to note that unlike for collisionless nondriven systems, the stationary state achieved by a driven one-component plasma does not depend on the initial particle distribution.

\section{COMPARISONS}

To compare the predictions of the theory with the results of the simulations, we consider the density and the temperature distributions inside the diode. The kinetic temperature is defined as

$$
k_{B} T(x)=\overline{v^{2}}(x)-\bar{v}^{2}(x),
$$

where the over bar denotes the velocity average at a given position $x$. The theoretical averages are calculated using the distribution function $f[\varepsilon(x, v)]$, while in the simulations, the averages are performed over the particle velocities within narrow bins along the $x$ axis. Note that because of the asymmetry of the velocity distribution at $x=0, T(0) \neq T_{c}$.

It is convenient to scale space and time with the diode length $L$ and the plasma frequency $\omega_{p}^{2} \equiv N e^{2} / \epsilon_{0} m L A$, respectively. Dimensionless coordinate and velocity can then be defined as $x^{*}=x / L$ and $v^{*}=v / L \omega_{p}$. In addition, Eqs. (4) and (7) show that adimensional temperature and adimensional voltage can be defined as $T^{*}=k_{B} T / m L^{2} \omega_{p}^{2}$ and $V^{*}$ $=e V / m L^{2} \omega_{p}^{2}$, respectively, and serve as the control parameters for the system.

In Fig. 1(a) the scaled temperature $T^{*}$ is plotted against the scaled coordinate $x^{*}$. We consider $T_{c}^{*}=0.05$ and also consider a device operating at its limiting current, $\dot{v}(x=0)=0$. A striking feature of this plot is that the temperature drops rapidly as one moves away from cathode toward anode. We next study the dependence of scaled density $n^{*}\left(x^{*}\right)=n(x) A L / N$ along the length of the diode. The density is very high near the cathode, where the average velocity is small. It then drops rapidly toward the anode, where particles are accelerated up to high speeds, see Fig. 1(b). Agreement between the simulations and the theory for both the kinetic temperature and density is excellent. Unlike the analytical solution, which only describes the stationary state of a driven onecomponent plasma, the simulations provide the entire dynamical evolution of the electronic system. They also show that the final stationary state is independent of the initial conditions, as discussed earlier.

We now study the current-voltage phase diagram of the device. In general, current is a function of the voltage drop, the temperature, the gap length, and the total charge of the device. However, by measuring the time in units of one over the plasma frequency $\omega_{p}^{-1}$, and the length in units of the gap length $L$, we can scale away two of these variables. The current density can be calculated using

$$
j=-e \int v f d v .
$$

Since in the steady state the current does not depend on either time or coordinate, integration along the $x$ axis and over the cross sectional area $A$ yields

$$
j L A=-e \int v f d v d x d^{2} r_{\perp} .
$$

Furthermore, since the current density is measured in units $[j] \sim[\mathrm{Nev} / \mathrm{AL}]$, rescaling it in terms of the gap length and the plasma frequency, we can write the reduced current density as $j^{*}=-e j / \varepsilon_{0} m L \omega_{p}^{3}$, which then satisfies

$$
j^{*}=\overline{\overline{v^{*}}}\left(V^{*}, T^{*}\right),
$$

where $\overline{\overline{v^{*}}}$ is the reduced velocity averaged over all the particles. The reduced average velocity, in turn, must be a function of the two previously introduced control parameters: the reduced voltage and temperature. Equation (11) is in fact a similarity transformation relating systems with different charge, length, temperature, and potential difference. In Fig. 2 we plot $j^{*}$ vs $V^{*}$ for various $T^{*}$. The phase diagram provides all the information about the current-voltage characteristics for all possible planar diodes. The first feature to note is that all the different curves emanate from the limiting current backbone, which traces a temperature dependent path in the $j^{*} \times V^{*}$ plane. To the left of the limiting current border, indicated by the solid line in Fig. 2, the distribution function can no longer be described by a unidirectional Maxwellian, such as expression (4). The transition resembles BoseEinstein condensation (BEC). In the case of BEC-below the critical temperature-a macroscopically populated ground state appears, and only a fraction of particles remains in the excited states. Similarly, in the case of our diode, to the left of the limiting curve, part of the charge must be expelled from the system before a stationary state can be achieved. As $T_{c}^{*} \rightarrow \infty$, the voltage effects become negligible 


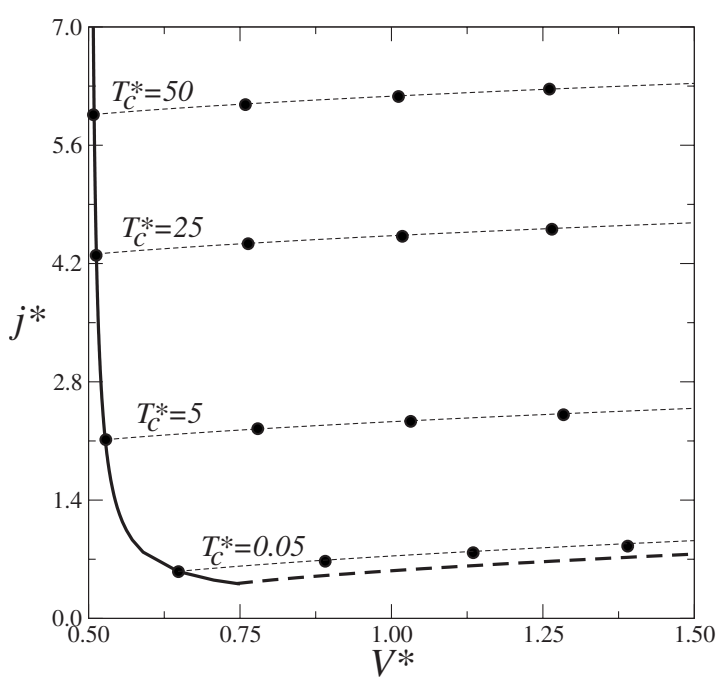

FIG. 2. Characteristic curves of $j^{*}$ vs $V^{*}$. The thick solid line represents the limiting current and the thick dashed line represents the zero-temperature limit. To the left of the solid curve, charge must be expelled from the system before a stationary state can be achieved. Dotted lines represent the theoretical results for the indicated temperatures. The circles are the results of the simulations at the same temperatures.

compared to the thermal ones, and the beam density becomes uniform across the gap. In this limit, it is possible to show that the dimensionless backbone curve asymptotes to a vertical line, $V^{*}=0.5$, Fig. 2 .

\section{CONCLUSIONS}

To conclude, we have studied the dynamics of a driven one-component plasma. Unlike the stochastic lattice gasses that are significantly abstracted from reality, the model studied in this paper is very similar to real electronic devices, such as the thermionic valves, diodes, and magnetrons. Furthermore, differently from the lattice gases whose dynamics is diffusive, the distribution function of collisionless systems satisfies the Vlasov equation. For the class of driven systems introduced in this paper, the stationary-state Vlasov equation can be solved exactly. The theory developed in this paper should, therefore, be relevant to the design and operation of real electronic devices.

It is important to stress that in the absence of collisions, a charged beam does not relax to an equilibrium with a known equation of state. In fact, the thermodynamic temperature is defined only in the vicinity of the hot emitting cathode. Away from the cathode, dynamics is controlled by the collisionless Vlasov equation, which has to be solved as a boundary-value problem. Once the solution is obtained, all the macroscopic quantities can be determined via appropriate averages. The kinetic temperature is found to vary strongly across the device gap, precluding the use of conventional isothermal or adiabatic assumptions and of the hydrodynamic formalisms.

\section{ACKNOWLEDGMENTS}

This work was supported by CNPq, FAPERGS, INCTFCx of Brazil, and by the Air Force Office of Scientific Research (AFOSR), USA, under Grant No. FA9550-09-1-0283.
[1] J. Marro and R. Dickman, Nonequilibrium Phase Transitions in Lattice Models (Cambridge University Press, New York, 1999).

[2] B. Derrida, E. Domany, and D. Mukamel, J. Stat. Phys. 69, 667 (1992).

[3] B. Derrida, S. A. Janowsky, J. L. Lebowitz, and E. R. Speer, J. Stat. Phys. 73, 813 (1993).

[4] L. Bertini, A. De Sole, D. Gabrielli, G. Jona-Lasinio, and C. Landim, J. Stat. Mech.: Theory Exp. (2007) P07014.

[5] D. Lynden-Bell, Mon. Not. R. Astron. Soc. 136, 101 (1967).

[6] T. Padmanabhan, Phys. Rep. 188, 285 (1990).

[7] P.-H. Chavanis and J. Sommeria, Mon. Not. R. Astron. Soc. 296, 569 (1998).

[8] T. M. Rocha Filho, A. Figueiredo, and M. A. Amato, Phys. Rev. Lett. 95, 190601 (2005).

[9] P.-H. Chavanis, Physica A 359, 177 (2006).

[10] A. Antoniazzi, D. Fanelli, J. Barré, P.-H. Chavanis, T. Dauxois, and S. Ruffo, Phys. Rev. E 75, 011112 (2007).

[11] Y. Levin, R. Pakter, and T. N. Teles, Phys. Rev. Lett. 100, 040604 (2008).

[12] Y. Levin, R. Pakter, and F. B. Rizzato, Phys. Rev. E 78, 021130 (2008).

[13] R. C. Davidson and H. Qin, Physics of Intense Charged Par- ticle Beams in High Energy Accelerators (World Scientific, Singapore, 2001).

[14] M. Reiser, Theory and Design of Charged Particle Beams (Wiley-Interscience, New York, 1994).

[15] G. H. Goedecke, B. T. Davis, C. Chen, and C. V. Baker, Phys. Plasmas 12, 113104 (2005).

[16] P. Martin and G. Donoso, Phys. Fluids B 1, 247 (1989).

[17] N. Jelić, R. Schrittwieser, and S. Kuhn, Phys. Lett. A 246, 318 (1998).

[18] H. Alfvén and P. Carlquist, Sol. Phys. 1, 220 (1967).

[19] S. S. Hasan and D. Ter Haar, Astrophys. Space Sci. 56, 89 (1978)

[20] M. A. Lieberman, C. Charles, and R. W. Boswell, J. Phys. D 39, 3294 (2006).

[21] W. Braun and K. Hepp, Commun. Math. Phys. 56, 101 (1977); A. Antoniazzi, F. Califano, D. Fanelli, and S. Ruffo, Phys. Rev. Lett. 98, 150602 (2007).

[22] Y. Levin, Rep. Prog. Phys. 65, 1577 (2002).

[23] J. D. Jackson, Classical Electrodynamics (John Wiley, New York, 1975).

[24] F. B. Rizzato, R. Pakter, and Y. Levin, Phys. Plasmas 14, 110701 (2007); R. Pakter and F. B. Rizzato, Phys. Rev. Lett. 87, 044801 (2001). 